\title{
Ischemic Conditioning Prevents Na,K-ATPase Dissociation from the Cytoskeletal Cellular Fraction after Repeat Renal Ischemia in Rats
}

\author{
CHRISTOPH AUFRICHT, BETTINA BIDMON, DAGMAR RUFFINGSHOFER, HEINZ REGELE, \\ KURT HERKNER, NORMAN J. SIEGEL, MICHAEL KASHGARIAN, AND SCOTT K. VAN WHY \\ Kinderdialyse [C.A., B.B., D.R.] and Ludwig Boltzmann Institute [H.R.], Departments of Pediatrics [C.A., \\ B.B., D.R., K.H.] and Pathology [H.R.], AKH Wien, A-1090 Vienna, Austria; and Departments of \\ Pediatrics and Pathology, Yale University School of Medicine, New Haven, Connecticut 06510, U.S.A.
}

[N.J.S., M.K., S.K.V.W.]

\begin{abstract}
ABST
Recent studies have suggested that heat shock proteins
(HSPs) are involved in the restoration of the cytoskeletal anchor-
age of Na,K-ATPase after renal ischemia. To determine their role
in ischemic conditioning, we investigated whether cytoskeletal
Na,K-ATPase was stabilized during repeat ischemia concurrent
with $25-\mathrm{kD}$ and 70 -kD HSPs induction. Anesthetized rats either
underwent single unilateral renal ischemia or were conditioned
with bilateral renal ischemia and, after $18 \mathrm{~h}$ of reflow, were then
subjected to repeat unilateral renal ischemia. Renal cortex was
harvested, and effects of single versus repeat ischemia were
compared by Triton X-100 extraction, by immunohistochemis-
try, and by an in vitro assay of Na,K-ATPase association with
isolated cytoskeletal fractions. In contrast to single ischemia,
repeat ischemia did not result in increased Triton X-100 extract-
ability of Na,K-ATPase. Levels of $25-\mathrm{kD}$ and $70-\mathrm{kD}$ HSPs were
significantly induced by ischemic conditioning and redistributed
\end{abstract}
Renal ischemia not only disrupts cellular homeostasis and causes injury but also induces a cellular stress response that participates in repair processes and may protect specific cellular structures against subsequent injury (1). Renal ischemia results in a transient relocation of $\mathrm{Na}, \mathrm{K}$-ATPase into the apical membrane domain in the proximal tubule cell $(2,3)$. This dislocated $\mathrm{Na}, \mathrm{K}$-ATPase remains functional but uses ATP to pump sodium back into the tubule lumen (2). Several clinical sequelae such as renal sodium loss and reduced glomerular filtration as a result of tubuloglomerular feedback can be explained by this redistribution of $\mathrm{Na}, \mathrm{K}-\mathrm{ATPase}$.

Received July 24, 2000; accepted February 22, 2001.

Correspondence and reprint requests: Christoph Aufricht, M.D., Kinderdialyse, Universitaetsklinik fuer Kinder und Jugendheilkunde, AKH Wien, Waehringer Guertel 18-20, A-1090 Vienna, Austria; e-mail: christoph.aufricht@akh-wien.ac.at

Supported by P12512-MED from the Austrian Science Foundation and by National Institute of Diabetes and Digestive Kidney Diseases grant DK 44336-06. S.K.V.W. is an Established Investigator of the American Heart Association.

DOI: $10.1023 / 01 . P D R .0000015128 .65800 .28$ into the cytoskeletal fraction after single and repeat ischemia. Immunohistochemistry also showed significant disruption of $\mathrm{Na}, \mathrm{K}-\mathrm{ATPase}$ within proximal tubules only after a single episode of ischemia, whereas repeat ischemia did not alter the pattern of restored $\mathrm{Na}, \mathrm{K}$-ATPase localization in conditioned renal cortex. The preserved association of Na,K-ATPase with the cytoskeletal fraction of conditioned renal cortex was effectively abolished in vitro by addition of antibodies against $25-\mathrm{kD}$ or $70-\mathrm{kD}$ HSP. These results suggest that $25-\mathrm{kD}$ and $70-\mathrm{kD}$ HSPs induced by ischemic conditioning stabilize the cytoskeletal anchorage of $\mathrm{Na}, \mathrm{K}-\mathrm{ATPase}$ during repeat renal ischemia. (Pediatr Res 51: 722-727, 2002)

Abbreviations
HSP, heat-shock protein
ECL, enhanced chemiluminescence

For $\mathrm{Na}, \mathrm{K}-\mathrm{ATPase}$ to be translocated, it must first dissociate from its cytoskeletal anchorage (2-5). Triton X-100 extraction fractionates the cellular pool of $\mathrm{Na}, \mathrm{K}$-ATPase into an insoluble pellet (cytoskeletal-associated fraction) and a soluble supernatant by simple differential centrifugation. The redistribution of $\mathrm{Na}, \mathrm{K}$-ATPase from pellet to supernatant has been used in several studies as a reproducible marker for ischemic injury of the proximal tubule cell. Using the same technique, the increased Triton X-100 extractability of Na,K-ATPase after renal ischemia was shown to be fully reversible during recovery at a rate dependent on the severity of the insult (3-5).

Recent studies have suggested that HSPs are involved in the restoration of the cytoskeletal anchorage of Na,K-ATPase (4-6). Renal ischemia, in vivo, rapidly induces the expression of $25-\mathrm{kD}$ and $70-\mathrm{kD}$ HSPs (4-8). The transient shift of $25-\mathrm{kD}$ HSP, a known actin-binding protein, into the Triton X-100insoluble pellet strongly suggests interactions with the disrupted cytoskeleton during the postischemic cellular reorganization $(6,9)$. The 70-kD HSPs, a family of molecular 
chaperons, show a distinct temporal pattern of postischemic cellular localization and intracellular distribution that coincides with the restoration of disrupted cytoskeletal elements during recovery of the proximal tubule cell from ischemia (8). Using functional in vitro assays, we recently demonstrated that enhanced 70-kD HSP activity and abundance results in stabilization of Na,K-ATPase in cytoskeletal subfractions $(4,5)$.

In the present study we sought to determine whether high cellular levels of $25-\mathrm{kD}$ and $70-\mathrm{kD}$ HSPs after pretreatment with renal ischemia (ischemic conditioning) might stabilize $\mathrm{Na}, \mathrm{K}-\mathrm{ATPase}$ in vivo during a subsequent ischemic injury. We compared the cellular localization and distribution of $\mathrm{Na}, \mathrm{K}$ ATPase after a single and after repeat ischemia in rat renal cortex, and evaluated potential functional interactions between these HSPs and Na,K-ATPase.

\section{METHODS}

The study was approved by the Institutional Animal Study Review Board. All experiments were performed in male Sprague-Dawley rats, weighing 225-300 g, anesthetized by i.p. thiobutabarbital sodium. Temperature was rectally monitored and sustained at normal values on a warming board throughout the anesthesia period. A polyethylene catheter was secured in an external jugular vein, and $5 \mathrm{~mL}$ of $0.9 \%$ saline was administered followed by an infusion of $1 \mathrm{~mL} / \mathrm{h}$. The abdomen was opened by a median incision, and the kidneys were exposed and, if macroscopically normal, carefully dissected. After anticoagulation with $125 \mathrm{IU}$ of heparin, renal ischemia was performed as previously described $(4,5)$. After the protocol, anesthetized animals were killed by i.v. or intracardial potassium chloride.

Bilateral ischemia (ischemic conditioning). Bilateral renal ischemia was accomplished by selective occlusion of the right renal artery and aorta just proximal to the left renal artery. After $45 \mathrm{~min}$ the clamps were removed, and reperfusion was visually confirmed. The abdominal incision was sutured, and the rats were allowed free access to food and water after recovery from anesthesia.

Unilateral ischemia (ischemic injury). Both preconditioned (18 h after bilateral ischemia) and nonpretreated rats were subjected to unilateral renal ischemia. The left kidney was removed immediately after induction of anesthesia, and the left renal pedicle was ligated to prevent exsanguination. Contralateral renal ischemia was accomplished by selective occlusion of the right renal artery. After $45 \mathrm{~min}$ the clamp was removed from the right kidney, and reperfusion was visually confirmed. After reflow of $15 \mathrm{~min}$ the right kidney was rapidly removed.

Triton X-100 extraction. Kidneys were decapsulated immediately after harvest on ice, and the renal cortex was homogenized in chilled extraction buffer (300-500 $\mathrm{mg}$ tissue in 15 $\mathrm{mL}$ ) containing $0.1 \%$ Triton X-100, $60 \mathrm{mM}$ 1,4-piperazinediethanesulfonic acid, $2 \mathrm{mM}$ 1,2-cyclohexylenedinitriloltriacetic acid, $1 \mathrm{mM}$ EDTA, $1 \mathrm{mM}$ EGTA, $100 \mathrm{mM} \mathrm{NaCl}, 0.5 \mathrm{mM}$ phenylmethylsulfonyl fluoride, $0.75 \mathrm{mg} / \mathrm{L}$ leupeptin, and 0.1 mM DTT using a Potter-Elvehjem homogenizer (Bellco Glass Co., Vineland, NJ, U.S.A.). The homogenate was centrifuged within $10 \mathrm{~min}$ at $35,000 \times \mathrm{g}$ for $14 \mathrm{~min}$ at $4^{\circ} \mathrm{C}$ to separate the
Triton X-100-soluble protein fraction from the insoluble cytoskeletal fraction. Protein fractions were saved at $-70^{\circ} \mathrm{C}$ until further analysis.

In three additional experiments, $100-\mu \mathrm{L}$ aliquots of isolated cytoskeletal pellet from renal cortex obtained either after single or after repeat renal ischemia were thawed on ice in $200 \mu \mathrm{L}$ of extraction buffer. In parallel, $100-\mu \mathrm{L}$ aliquots of cytoskeletal pellets isolated after repeat renal ischemia were incubated in $200 \mu \mathrm{L}$ of buffer with $50 \mu \mathrm{g}$ of anti $25-\mathrm{kD}$ HSP antibody, with $50 \mu \mathrm{g}$ of anti 70-kD HSP antibody, or with both antibodies (Stressgen Biotechnologies Corp., Victoria, BC, Canada). These mixtures were resuspended and incubated for $20 \mathrm{~min}$ in room temperature. Differential centrifugation was then repeated at $35,000 \times g$ for $14 \mathrm{~min}$ at $4^{\circ} \mathrm{C}$. Supernatants, containing the dissociated proteins, were saved at $-70^{\circ} \mathrm{C}$ until further analysis.

Western blot analysis. Protein concentrations were assessed in duplicate by Bradford analysis. Protein samples were electrophoresed through SDS-polyacrylamide gels and electrophoretically transferred to nitrocellulose $(4,5)$. Membranes were incubated with the primary antibodies against 70-kD HSP (Sigma Chemical Co., St. Louis, MO, U.S.A.), 25-kD HSP (SPA801; Stressgen Biotechnologies), and alpha-subunit of Na,K-ATPase (Upstate Biotechnology, Lake Placid, NY, U.S.A.). Detection was accomplished with secondary antibodies (Sigma Chemical Co.) and ECL using ECL Western blotting analysis system and protocols (Renaissance; PerkinElmer Life Science Products, Boston, MA, U.S.A.).

Densitometric analysis was performed by image analysis software (Image Master Analysis Software; Amersham Pharmacia Biotech, Uppsala, Sweden). For analysis of Western blots, calibration curves were constructed to determine the linearity of the ECL detection system using serial loading of cellular protein fractions. Light emission was proportional to protein loading in the range of 3.75 to $15 \mu \mathrm{g}$ of a given sample, indicating that 4 -fold changes were within the linear range.

Cellular distribution. As previous studies have shown that approximately two thirds of total cellular proteins are recovered in the Triton X-100-soluble fraction, the insoluble pellet was resuspended in half the volume of the original homogenate, resulting in approximately equal protein concentrations in both cellular fractions (approximately $4-6 \mu \mathrm{g} / \mu \mathrm{L})(4-6)$. Routine use of protein loading of $7.5 \mu \mathrm{g}$ was sufficient for analysis of HSP distribution between the soluble and insoluble fraction and for changes of Na,K-ATPase distribution. Induction of HSPs was densitometrically assessed after differential protein loading, comparing $15 \mu \mathrm{g}$ of nonpretreated samples with $3.75 \mu \mathrm{g}$ of pretreated samples. One more dilution $(20 \mu \mathrm{g}$ versus $2 \mu \mathrm{g}$ ) was added for 70-kD HSP induction.

Specific immunodensitometric signals at three different exposures in the linear range of the protein-to-signal intensity relationship were determined from nine rats in each experimental group and compared with an internal standard (for HSP, pooled ischemia-conditioned cortex; for Na,K-ATPase, pooled nonpretreated cortex). Final data for Na,K-ATPase and HSP distribution between soluble and insoluble cellular fractions and HSP induction were individually computed for each renal 
cortex after correction for protein distribution between the different cellular fractions after Triton X-100 extraction.

Immunocytochemical studies. A separate set of animals underwent the same conditioning and ischemic injury along with parallel controls. Renal cortical slices of approximately $2.0 \mathrm{~mm}$ were incubated in paraformaldehyde fixative. The fixed slices were embedded in paraffin and sectioned at $4 \mu \mathrm{m}$. After blocking of nonspecific binding sites, the identical primary antibodies to Na,K-ATPase as in Western blot analysis were applied in PBS $0.5 \%$ BSA for a total of $1 \mathrm{~h}$. After repeated washes, the sections were then exposed to antimouse $\mathrm{MAb}$ conjugated to horseradish peroxidase in an equivalent procedure to the primary antibody. The sections were evaluated on a Zeiss microscope (Axiophot, Zeiss, Germany).

Data analysis. ANOVA or Wilcoxon signed-rank test, as appropriate, was used. Values for each reflow interval were compared with their respective controls and considered to be significantly different if $p<0.05$. Changes are expressed as mean and $95 \%$ confidence intervals.

\section{RESULTS}

In the first part of this study we analyzed whether ischemic conditioning results in increased levels of cytoskeletally associated $25-\mathrm{kD}$ and $70-\mathrm{kD}$ HSPs in rat renal cortex after repeat renal ischemia (Figs. 1 and 2). In nonpretreated renal cortex, unilateral ischemia resulted in a significant translocation of $25-\mathrm{kD}$ and $70-\mathrm{kD}$ HSPs into the cytoskeletal cellular fraction, shifting the ratio of Triton X-100-soluble to -insoluble from 4.2 to 1.2 for $25-\mathrm{kD}$ HSP, and from 6.3 to 1.4 for $70-\mathrm{kD}$ HSP. Pretreatment with bilateral renal ischemia resulted in a significantly increased abundance of both HSPs at $18 \mathrm{~h}$ of recovery, the majority detected in the Triton X-100-soluble cellular fraction. At that time, repeat renal ischemia again resulted in a significant translocation of HSPs into the cytoskeletal pellet, shifting the ratio of Triton X-100-soluble to -insoluble from 5.1 to 2.1 for $25-\mathrm{kD}$ HSP, and from 8.1 to 2.5 for $70-\mathrm{kD}$ HSP.
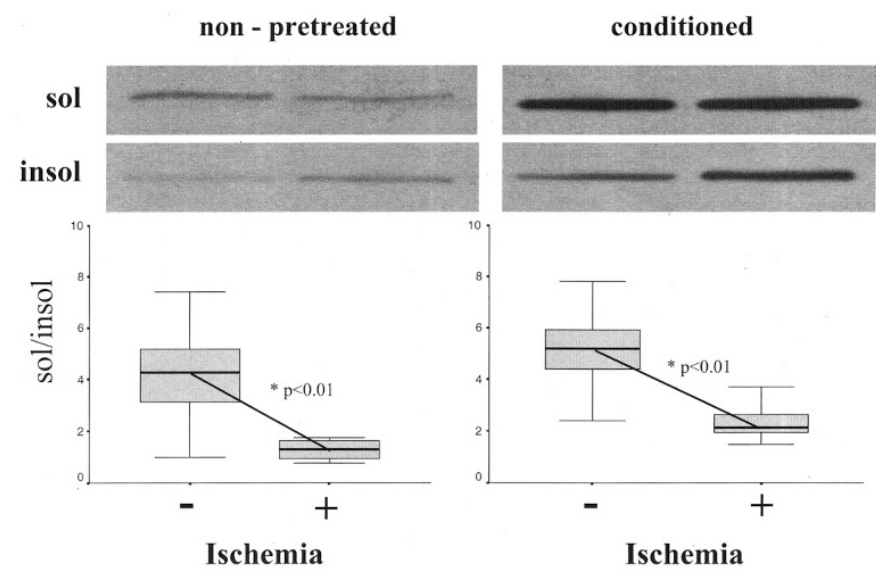

Figure 1. Representative immunoblot and densitometry $(n=9)$ of Triton X-100-soluble and -insoluble 25-kD HSPs in rat renal cortex. In nonpretreated animals $(l e f t)$, unilateral renal ischemia $(+)$ resulted in a shift of $25-\mathrm{kD}$ HSPs to the cytoskeletal pellet. In animals with ischemic conditioning (right), the $25-\mathrm{kD}$ HSPs were markedly elevated. Repeat ischemia $(+)$ resulted again in a shift of $25-\mathrm{kD}$ HSPs to the cytoskeletal pellet.
Ischemia
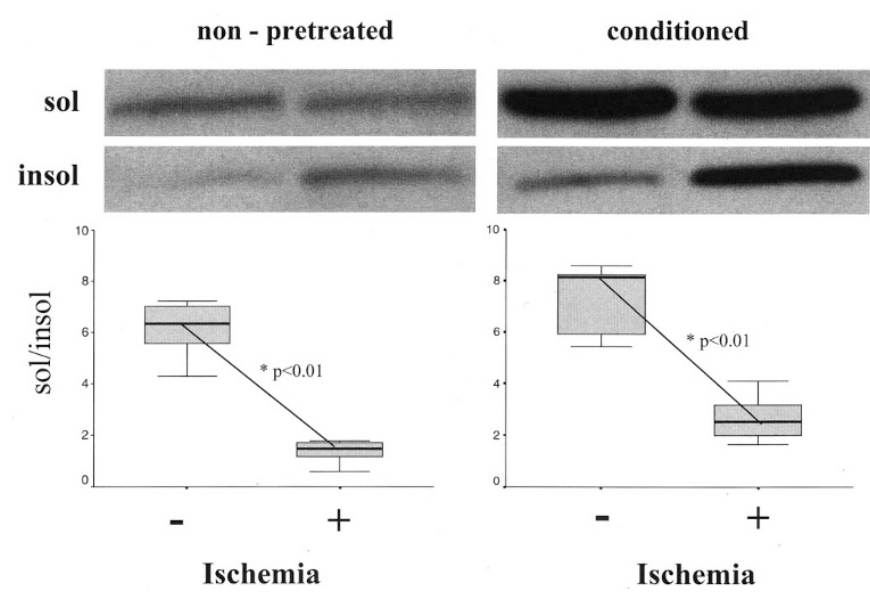

Figure 2. Representative immunoblot and densitometry $(n=9)$ of Triton $\mathrm{X}-100-$ soluble and -insoluble $70-\mathrm{kD}$ HSPs in rat renal cortex. In nonpretreated animals (left), unilateral renal ischemia $(+)$ resulted in a shift of $70-\mathrm{kD}$ HSPs to the cytoskeletal pellet. In animals with ischemic conditioning (right), the 70-kD HSPs were markedly elevated. Repeat ischemia (+) resulted again in a shift of $70-\mathrm{kD}$ HSPs to the cytoskeletal pellet.

In combination with the marked induction of total cellular HSPs after ischemic pretreatment (7.5-fold for 25-kD HSP and 19.5-fold for $70-\mathrm{kD}$ HSP) this cellular redistribution after repeat ischemia resulted in high levels of cytoskeletalassociated $25-\mathrm{kD}$ and $70-\mathrm{kD}$ HSPs in conditioned rat renal cortex.

In the second part of the study, we analyzed whether ischemic conditioning stabilizes the cytoskeletal association of $\mathrm{Na}, \mathrm{K}-\mathrm{ATPase}$ in rat renal cortex after repeat renal ischemia (Figs. 3 and 4). In nonpretreated animals, renal ischemia resulted in a marked increase of Triton X-100-extractable $\mathrm{Na}, \mathrm{K}-\mathrm{ATPase}$ at $15 \mathrm{~min}$ of reflow (1.8-fold increase (1.4-2.2; $p<0.01$ ). In conditioned animals (at $18 \mathrm{~h}$ of reflow) Triton
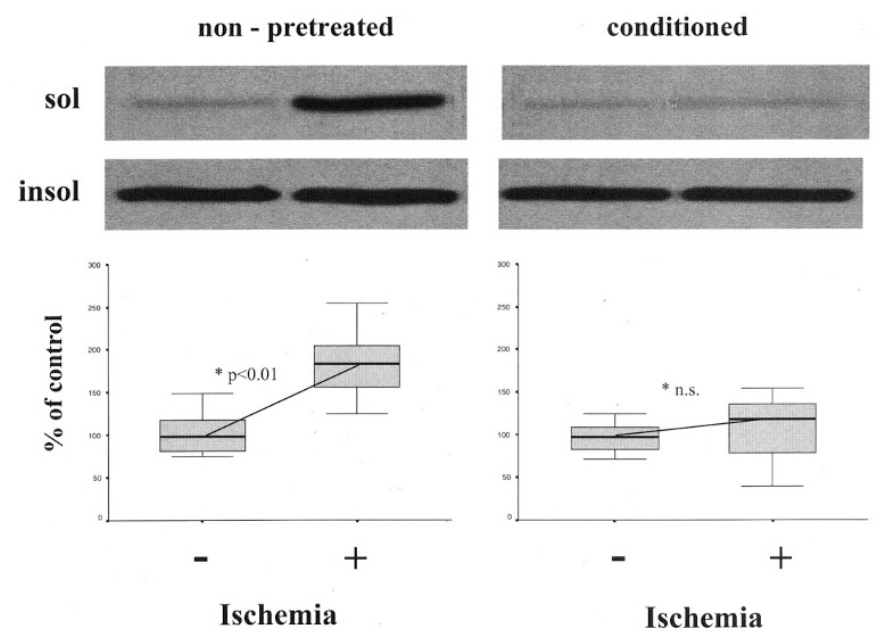

Figure 3. Representative immunoblot and densitometry $(n=9)$ of Triton $\mathrm{X}-100$-soluble and -insoluble Na,K-ATPase in rat renal cortex. In nonpretreated animals (left), unilateral renal ischemia $(+)$ resulted in a marked increase of Triton X-100-soluble Na,K-ATPase. In animals with ischemic conditioning (right), Triton X-100-soluble Na,K-ATPase had returned to basal levels. After repeat ischemia there was no significant increase in Triton X-100 extractability of Na,K-ATPase $(+)$. Data are expressed as percentage of the specific signal from pooled renal cortex from nonpretreated rats. 

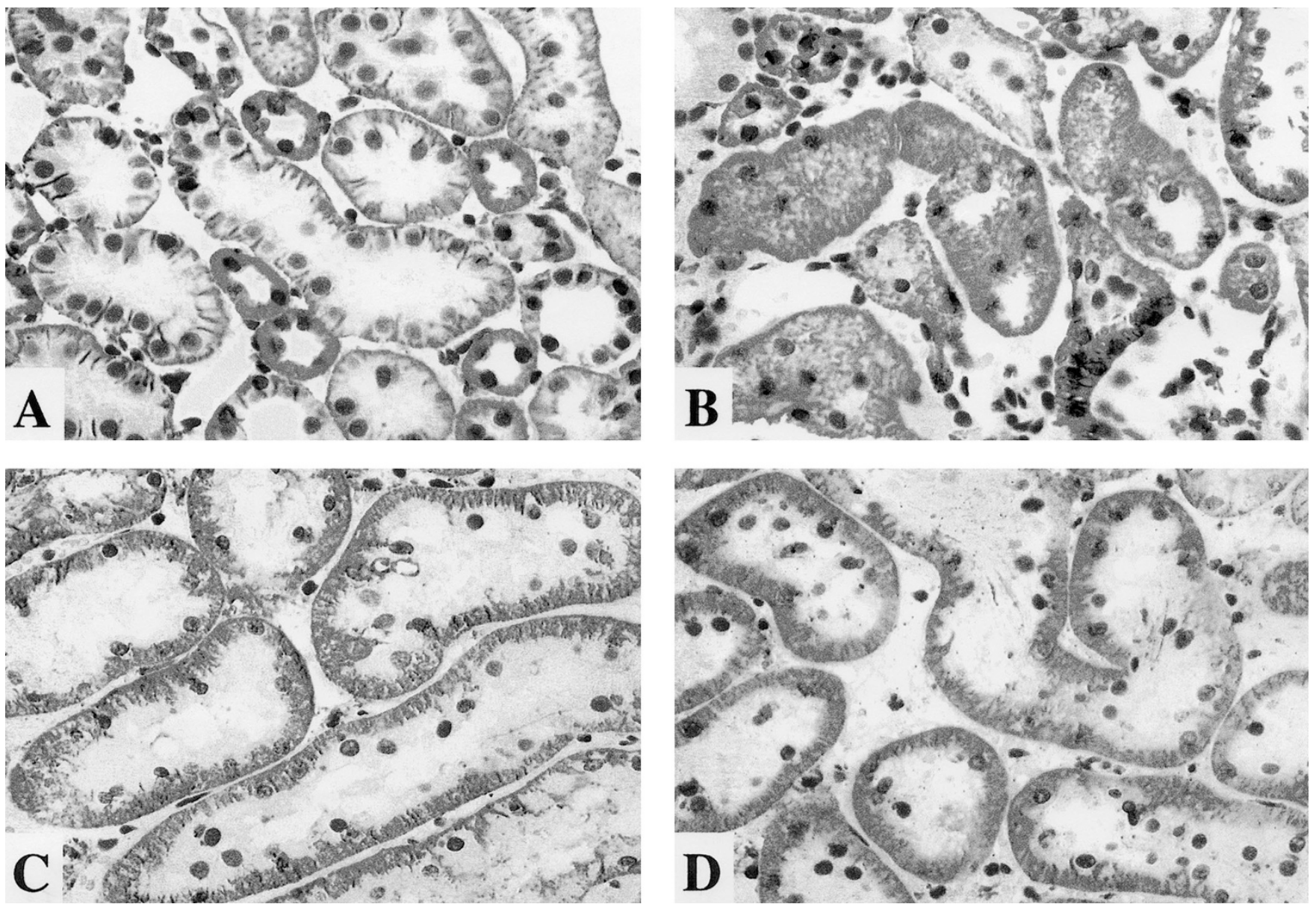

Figure 4. The pattern of immunolocalization of $\mathrm{Na}, \mathrm{K}-\mathrm{ATPase}$ was compared in proximal tubular cells of nonpretreated rats $(A, B)$ and of ischemia-conditioned rats $(C, D)$ between renal cortex harvested before $(A, C)$ or after $(B, D)$ ischemia. The staining patterns suggest that the Na,K-ATPase localization is stabilized during repeat ischemia in conditioned rat renal cortex.

X-100-extractable Na,K-ATPase had returned to baseline levels. Repeat renal ischemia resulted in no significant increase in Triton X-100 extractability of Na,K-ATPase (0.8-1.4). Reduction in Triton X-100-insoluble cytoskeletal Na,K-ATPase did not reach statistical significance after single or repeat ischemia (Fig. 3).

This stabilization of Na,K-ATPase localization in conditioned renal cortex was also found by immunohistochemistry (Fig. 4). In proximal tubule cells of nonpretreated rat renal cortex, renal ischemia resulted in obvious dislocation of $\mathrm{Na}, \mathrm{K}-$ ATPase from the basolateral domain into a diffuse cytoplasmic pattern with an overall decreased signal. In proximal tubule cells of conditioned renal cortex (at $18 \mathrm{~h}$ of reflow), $\mathrm{Na}, \mathrm{K}$ ATPase had reaccumulated in the basolateral domain. Repeat ischemia resulted in no discernible disruption of this staining pattern. The pattern of tubule Na,K-ATPase distribution remained unchanged in conditioned renal cortex after the second ischemic injury, when compared with the pattern in the contralateral conditioned kidneys, which did not undergo repeat ischemia.

In the final part of this study, we analyzed for functional interactions between $25-\mathrm{kD}$ and $70-\mathrm{kD}$ HSPs and stabilization of $\mathrm{Na}, \mathrm{K}-\mathrm{ATPase}$ in cytoskeletal fractions of rat renal cortex isolated after ischemic conditioning (Fig. 5). The differential

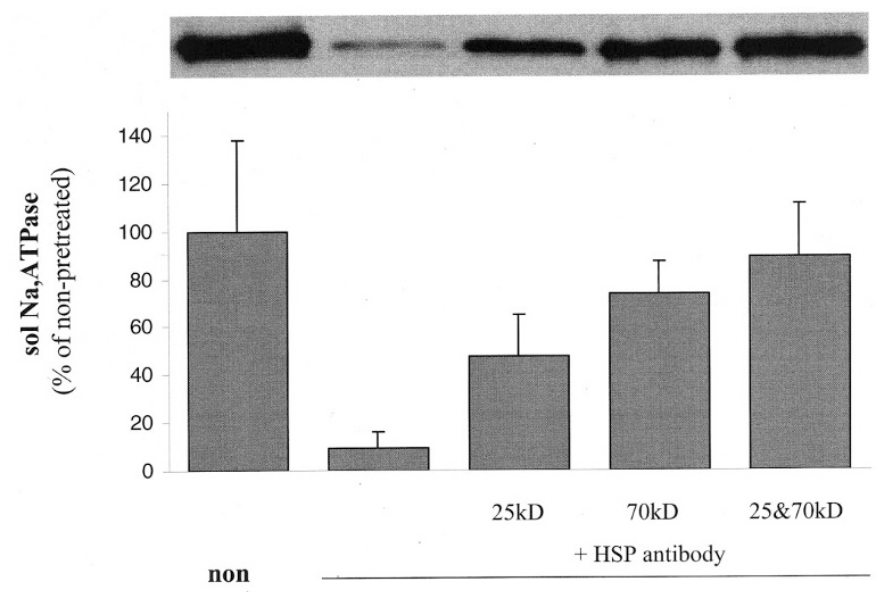

pretreated conditioned

Figure 5. Representative immunoblot and densitometry of differential Triton X-100 extractability of Na,K-ATPase from isolated cytoskeletal associated fractions $(n=3)$. Isolated cytoskeletal pellets obtained from rat renal cortex treated either by single (nonpretreated) or by repeat renal ischemia (conditioned) were incubated with anti-25-kD or anti-70-kD HSP antibody, or both. After the incubation interval, repeat Triton X-100 extraction was performed. Cytoprotective effects of ischemic conditioning (as demonstrated by stabilization of $\mathrm{Na}, \mathrm{K}$-ATPase in the cytoskeletal pellet isolated after repeat renal ischemia) were reversed by addition of anti-HSP antibodies. 
extractability of $\mathrm{Na}, \mathrm{K}-\mathrm{ATPase}$ from cytoskeletal fractions isolated from renal cortex either after single or after repeat ischemia was reproduced in a functional in vitro assay. Parallel incubation and repeat Triton X-100 extraction of isolated cytoskeletal protein fractions demonstrated significant stabilization of $\mathrm{Na}, \mathrm{K}-\mathrm{ATPase}$ in the conditioned cytoskeletal fraction.

We then found direct evidence for functional interactions between the HSPs and the cytoskeletal fraction associated with stabilization of $\mathrm{Na}, \mathrm{K}-\mathrm{ATPase}$ in this fraction during ischemic conditioning. Addition of antibodies against $25-\mathrm{kD}$ or $70-\mathrm{kD}$ HSPs attenuated stabilization of Na,K-ATPase in the cytoskeletal fraction (Fig. 5). Incubation of conditioned cytoskeletal pellets with extraction buffer enriched with antibodies against $25-\mathrm{kD}$ or $70-\mathrm{kD}$ HSPs resulted in markedly increased Triton X-100 extractability of Na,K-ATPase. The effect of adding antibody against both HSPs was additive and resulted in complete elimination of preserved $\mathrm{Na}, \mathrm{K}-\mathrm{ATPase}$ association with the cytoskeletal fraction in the preconditioned pellets.

\section{DISCUSSION}

Our study demonstrates that in vivo ischemic conditioning prevents dissociation of $\mathrm{Na}, \mathrm{K}-\mathrm{ATPase}$ from its cytoskeletal anchorage in rat renal cortex after repeat ischemia. This in vivo stabilization of $\mathrm{Na}, \mathrm{K}-\mathrm{ATPase}$ was associated with marked induction and cytoskeletal redistribution of $25-\mathrm{kD}$ and $70-\mathrm{kD}$ HSPs in conditioned renal cortex. Incubation of isolated cytoskeletal fractions of conditioned renal cortex with blocking antibodies against $25-\mathrm{kD}$ or $70-\mathrm{kD}$ HSPs abolished this stabilization of $\mathrm{Na}, \mathrm{K}-\mathrm{ATPase}$ in vitro.

Renal ischemia is known to cause transient disruption of the cellular localization and distribution of $\mathrm{Na}, \mathrm{K}-\mathrm{ATP}$ ase in the proximal tubular cell after nonlethal injury $(3,10)$. For Na,KATPase to be translocated from its basolateral membrane domain, it must first be detached from its cytoskeletal anchorage, which has been defined by Triton X-100 extractability (2, $3)$. In agreement with previous reports, this study shows that Triton X-100-extractable Na,K-ATPase was increased after single renal ischemia and had returned to basal levels in renal cortex after bilateral ischemia and $18 \mathrm{~h}$ of reflow. This restoration of the cytoskeletal association of Na,K-ATPase has been shown to occur by recycling of displaced elements rather than by their increased biosynthesis $(1,10)$.

The HSPs are ideal candidates for such posttranslational repair mechanisms $(11,12)$. $25-\mathrm{kD}$ HSPs are known to bind to the actin cytoskeleton, and 70-kD HSPs bind to hydrophobic sites of disrupted proteins (12). From these established functions of $25-\mathrm{kD}$ and $70-\mathrm{kD}$ HSPs, one would predict a transiently increased affinity of both HSPs for the Triton X-100insoluble cellular fraction of rat renal cortex isolated after ischemic injury. In this study, we confirm that renal ischemia rapidly induces the elaboration of $25-\mathrm{kD}$ and $70-\mathrm{kD}$ HSPs $(4$, $6-8$ ). Our data further demonstrate that in addition to $25-\mathrm{kD}$ HSPs, 70-kD HSPs also translocate into the cytoskeletal cellular fraction after renal ischemia (6). Moreover, these results extend the role of $25-\mathrm{kD}$ and $70-\mathrm{kD}$ HSPs from postischemic repair to potential cytoprotection against repeat ischemia, as not only single but also repeat renal ischemia resulted in a marked cytoskeletal shift of both HSPs. As ischemic conditioning potently increased the expression levels of these inducible HSPs but did not alter their pattern of postischemic redistribution, repeat ischemia resulted in high levels of $25-\mathrm{kD}$ and $70-\mathrm{kD}$ HSPs in the cytoskeletal fraction of cellular proteins.

This fraction is known to contain the actin cytoskeleton, $\mathrm{Na}, \mathrm{K}-\mathrm{ATPa} e$, and other cytoskeletal-associated proteins that are able to bind to each other (3-7). Stabilization by capping proteins like 25-kD HSP or molecular chaperons such as $70-\mathrm{kD}$ HSP may therefore result in improved binding and stabilization of the cytoskeletal anchorage of $\mathrm{Na}, \mathrm{K}$-ATPase $(4-6,12)$. Our data support this hypothesis as repeat renal ischemia did not cause a repeat increase of Triton X-100-extractable Na,KATPase in the conditioned renal cortex. Furthermore, the immunohistochemical findings are consistent with the detergent extractability data. In agreement with previous studies, the cellular distribution of $\mathrm{Na}$,K-ATPase was basolateral in proximal tubular cells in renal cortex harvested from control animals and was more diffusely distributed throughout the cell after single renal ischemia (10). In renal cortex harvested after $18 \mathrm{~h}$ of reflow (ischemic conditioning) the tubules display some typical postischemic changes including cellular dropout with loss of tubular integrity; however, the remaining proximal tubule cells show that basolateral localization of Na,K-ATPase is restored at this interval. Repeat ischemia after such ischemic conditioning, at high cellular abundance of $25-\mathrm{kD}$ and $70-\mathrm{kD}$ HSPs, resulted in no repeat disruption of the basolateral distribution of Na,K-ATPase. Using colocalization studies, we recently found close physical proximity between 25-kD HSPs and disrupted (DNAse-reactive) actin fragments after renal ischemia (6). Similar postischemic colocalization was found between the molecular chaperon 70-kD HSP and disrupted, displaced Na,K-ATPase (13). These temporal and spatial associations suggest that in vivo stabilization of Na,K-ATPase after ischemic conditioning might at least in part be mediated by high levels of cytoskeletally associated $25-\mathrm{kD}$ and $70-\mathrm{kD}$ HSPs.

Using a similar in vivo protocol Zager and Baltes (14) have previously described that rat kidneys exhibited comparable morphologic injury after single and repeat ischemia, and that repeat ischemia did not prolong overall postischemic recovery of renal function. In contrast, heat pretreatment did not protect morphology or global function against experimental renal ischemia in isolated perfused kidneys or proximal tubules, despite comparable induction of 70-kD HSP $(15,16)$. Interestingly, however, heat-conditioned kidneys demonstrated significant preservation of sodium reabsorption (15). As the authors did not assess cellular localization or distribution of Na,K-ATPase in proximal tubule cells, it could be that the heat pretreatment resulted in an unappreciated preservation of Na,K-ATPase polarity and function, thus preserving sodium reabsorptive ability. HSP-mediated stabilization of the cytoskeletal anchorage and localization of Na,K-ATPase could be the underlying mechanism. Consistent with this concept, heat conditioning of cultured renal cells resulted in induction of 70-kD HSPs and preservation of cytoskeletal structure against subsequent injury 
by energy depletion (17). Similarly, selective overexpression of 25-kD HSPs protected the actin-based cytoskeleton against disruption by subsequent injurym and transfection of proximal tubular cells with 70-kD HSPs resulted in cytoprotection against heat $(9,18)$. Thus, findings from other in vivo and in vitro systems also corroborate that effects of $25-\mathrm{kD}$ and $70-\mathrm{kD}$ HSPs might mediate cytoprotection conferred by conditioning treatments.

In search of direct evidence for HSP-mediated restoration of the cytoskeletal anchorage of $\mathrm{Na}, \mathrm{K}-\mathrm{ATPase}$ after in vivo renal ischemia, we recently have developed a functional in vitro repair assay (5). In this system, coincubation of cytoskeletal and associated proteins obtained during early reflow after renal ischemia (exhibiting severe injury of the cytoskeletal anchorage of Na,K-ATPase) and noncytoskeletal proteins obtained during later reflow (showing high HSP expression) resulted in specific translocation of 70-kD HSPs from the noncytoskeletal supernatant into the cytoskeletal pellet, functionally associated with dose-dependent stabilization of $\mathrm{Na}, \mathrm{K}$-ATPase within this cytoskeletal fraction. This stabilization was reproduced by purified HSP and abolished by anti-HSP antibodies (5). In the present study, we modified this in vitro assay to investigate the role of $25-\mathrm{kD}$ and $70-\mathrm{kD}$ HSPs in the stabilization of $\mathrm{Na}, \mathrm{K}-$ ATPase after ischemic conditioning. Based on our previous findings, we expected that high abundance of HSPs would stabilize whereas blocking of HSPs would disrupt the association of Na,K-ATPase with isolated cytoskeletal fractions. As expected, $\mathrm{Na}, \mathrm{K}$-ATPase was stabilized in cytoskeletal fractions of conditioned renal cortex (isolated at high abundance of HSPs). This stabilization was almost completely abolished in vitro by incubation with antibodies simultaneously against 25-kD and 70-kD HSPs. Incubation with antibody against an individual HSP attenuated the effect. As the effects of each antibody were additive, the findings are consistent with different, but complementary, functions of the actin-binding $25-\mathrm{kD}$ HSP and the molecular chaperon $70-\mathrm{kD}$ HSP. These in vitro results provide direct evidence for essential roles of both HSPs in the cytoprotection against repeat renal ischemia after ischemic conditioning.

Taken together, this study confirms the concordant induction of the $25-\mathrm{kD}$ and $70-\mathrm{kD}$ HSPs after renal ischemia. It further shows increased association of these HSPs with the cytoskeletal fraction after single or repeat renal ischemia. In contrast to single ischemia, repeat ischemia at high cellular abundance of $25-\mathrm{kD}$ and $70-\mathrm{kD}$ HSPs did not result in disruption of the cellular distribution or localization of Na,K-ATPase. The stabilization of Na,K-ATPase interactions with the cytoskeleton could be reversed in vitro by antibodies against $25-\mathrm{kD}$ and 70-kD HSPs. This study thus supports the concept that ischemic conditioning protects the integrity of the cytoskeletal anchorage of $\mathrm{Na}, \mathrm{K}-\mathrm{ATPase}$ after repeat renal ischemia by HSP-mediated mechanisms.

\section{REFERENCES}

1. Siegel NJ, Devarajan P, Van Why SK 1994 Renal cell injury: metabolic and structural alterations. Invited review. Pediatr Res 36:129-136

2. Molitoris BA $1991 \mathrm{New}$ insights into the cell biology of ischemic acute renal failure. J Am Soc Nephrol 1:1263-1270

3. Molitoris BA, Dahl R, Geerdes A 1992 Cytoskeletal disruption and apical redistribution of proximal tubule Na,K-ATPase during ischemia. Am J Physiol 263:F488F495

4. Aufricht C, Lu E, Thulin G, Kashgarian M, Siegel NJ, Van Why SK 1998 ATP releases HSP-72 from protein aggregates after renal ischemia. Am J Physiol 274:F268-F274

5. Bidmon B, Endemann M, Mueller T, Arbeiter K, Herkner K, Aufricht C 2000 HSP-70 repairs tubule cell structure after renal ischemia. Kidney Int 58:2400-2407

6. Aufricht C, Ardito T, Thulin G, Kashgarian M, Siegel NJ, Van Why SK 1998 Heat-shock protein 25 induction and redistribution during actin reorganization after renal ischemia. Am J Physiol 274:F215-F222

7. Schober A, Mueller E, Thurau K, Beck FX 1997 The response of heat shock proteins 25 and 72 to ischaemia in different kidney zones. Pflugers Arch 434:292-299

8. Van Why SK, Hildebrand F, Ardito T, Mann AS, Siegel NJ, Kashgarian M 1992 Induction and intracellular localization of HSP-72 after renal ischemia. Am J Physiol 263:F769-F775

9. Huot J, Houle F, Spitz DR, Landry J 1996 HSP27 phosphorylation-mediated resistance against actin fragmentation and cell death induced by oxidative stress. Cancer Res 56:273-279

10. Van Why SK, Mann AS, Ardito T, Siegel NJ, Kashgarian M 1994 Expression and molecular regulation of $\mathrm{Na}+-\mathrm{K}+$-ATPase after renal ischemia. Am J Physiol 267:F75-F85

11. Minowada G, Welch WJ 1995 Clinical implications of the stress response. J Clin Invest 95:3-12

12. Morimoto RI, Tissieres A, Georgopoulos C 1994 The Biology of Heat Shock Proteins and Molecular Chaperones. Cold Spring Harbor Laboratory Press, New York

13. Van Why SK, Siegel NJ 2001 Heat shock proteins: role in prevention and recovery from acute renal failure. In: Molitoris B, Finn W (eds) Acute Renal Failure: A Companion to Brenner \& Rector's The Kidney. WB Saunders, Philadelphia, pp 143-156

14. Zager RA, Baltes LA 1984 Responses of the ischemic acute renal failure kidney to additional ischemic events. Kidney Int 26:689-700

15. Joannidis M, Cantley LG, Spokes K, Medina R, Pullman J, Rosen S, Epstein FH 1995 Induction of heat-shock proteins does not prevent renal tubular injury following ischemia. Kidney Int 47:1752-1759

16. Zager RA, Iwata M, Burkhart KM, Schimpf BA 1994 Post ischemic acute renal failure protects proximal tubules from $\mathrm{O}_{2}$ deprivation, possibly by inducing uremia. Kidney Int 45:1760-1768

17. Borkan SC, Wang YH, Lieberthal W, Burke PR, Schwartz JH 1997 Heat stress ameliorates ATP depletion-induced sublethal injury in mouse proximal tubule cells. Am J Physiol 272:F347-F355

18. Turman MA, Rosenfeld SL 1999 Heat shock protein 70 overexpression protects LLC-PK1 tubular cells from heat shock but not hypoxia. Kidney Int 55:189-97 